\title{
TASK-1 induces gefitinib resistance by promoting cancer initiating cell formation and epithelial-mesenchymal transition in lung cancer
}

\author{
XING-GUANG WANG ${ }^{1}$, NA-XIN YUAN ${ }^{2},{\text { XIN-PENG } \text { LI }^{2} \text { and FANG-FANG CHEN }}^{3}$ \\ ${ }^{1}$ Department of Respiratory Medicine, Shandong Provincial Hospital Affiliated to Shandong University, \\ Jinan, Shandong 250021; ${ }^{2}$ Department of Respiratory Medicine, Dezhou People's Hospital, Dezhou, \\ Shandong 253014; ${ }^{3}$ Department of Respiratory Medicine, Shandong Provincial Qianfoshan Hospital \\ Affiliated to Shandong University, Jinan, Shandong 250014, P.R. China
}

Received June 23, 2016; Accepted April 28, 2017

DOI: $10.3892 /$ etm.2017.5426

\begin{abstract}
Cancer initiating cell (CIC) formation and epithelial-mesenchymal transition (EMT) are pivotal events in lung cancer cell invasion and metastasis. They have been shown to occur in gefitinib resistance. Studying the molecular mechanisms of CICs, EMT and acquired gefitinib resistance will enhance the understanding of the pathogenesis and progression of the disease and offer novel targets for effective therapies. TWIK-related acid-sensitive $\mathrm{K}(+)$ (TASK-1) is expressed in a subset of non-small-cell lung cancer (NSCLC) cell lines, where it promotes cell proliferation while inhibiting apoptosis. In the present study, TASK-1 was demonstrated to induce gefitinib resistance in the A549 NSCLC cell line. Overexpression of TASK-1 promoted the acquisition of CIC-like traits by A549 cells. CD133, octamer-binding transcription factor 4 (OCT-4) and Nanog have been suggested to be markers of CICs in lung cancer. It was demonstrated that overexpression of TASK-1 promoted CD133, OCT-4 and Nanog protein expression in A549 cells. Increased formation of stem cell-like populations results in EMT of cancer cells. The present study found that overexpression of TASK-1 promoted EMT of A549 cells. Thus, downregulation of TASK-1 may represent a novel strategy for EMT reversal, decreasing CIC-like traits and increasing gefitinib sensitivity of NSCLCs.
\end{abstract}

Correspondence to: Dr Fang-Fang Chen, Department of Respiratory Medicine, Shandong Provincial Qianfoshan Hospital Affiliated to Shandong University, 16766 Jingshi Road, Jinan, Shandong 250014, P.R. China

E-mail: chenfangfang16766@163.com

Key words: cancer initiating cells, epithelial-mesenchymal transition, lung cancer, TASK-1

\section{Introduction}

Lung cancer is a heterogeneous malignancy with aggressive phenotypes $(1,2)$. Non-small-cell lung cancer (NSCLC) accounts for $\sim 85 \%$ of lung cancer cases (2). Lung cancers harboring somatic mutations in exons encoding the tyrosine kinase domain of the epidermal growth factor receptor (EGFR) exhibit a significant tumor regression when treated with the EGFR tyrosine kinase inhibitors (TKIs) gefitinib or erlotinib in $\sim 70 \%$ of cases (3-5). However, acquired resistance inevitably develops in an overwhelming majority of these patients.

Epithelial to mesenchymal transition (EMT) is associated with the acquired resistance of NSCLC to gefitinib $(6,7)$. It is a process by which cells undergo a morphological shift from the epithelial polarized to the mesenchymal fibroblastoid phenotype. EMT has been recognized to have pivotal roles in several diverse processes during embryonic development, chronic inflammation and fibrosis, as well as tumor progression (8-11). During EMT, epithelial cells lose their defined cell-cell/cell-substratum contacts and their structural/functional polarity, and become spindle-like.

Lung cancer stem cells (CSCs) or cancer-initiating cells (CICs) have been identified and demonstrated to constitute a primitive cell population capable of self-renewal and differentiation that have the unique capacity to give rise to new tumors upon serial transplantation (12-15). They represent a small population of undifferentiated tumorigenic cells responsible for tumor initiation, maintenance and spreading. Resistance to conventional chemotherapeutic drugs is a common characteristic of CICs (16). It has been reported that lung CICs were associated with gefitinib resistance (17).

TWIK-related acid-sensitive $\mathrm{K}(+)$ (TASK-1) is expressed in a subset of NSCLCs, where it is functional, and promoted the proliferation and inhibited apoptosis in a highly TASK-1-expressing lung cancer cell line (18). The present study demonstrated that TASK-1 induced gefitinib resistance in NSCLC A549 cells. Overexpression of TASK-1 promoted the formation of CICs in A549 cells. CD133, octamer-binding transcription factor 4 (OCT-4) and Nanong have been proposed as markers of CICs in lung cancer $(13,19,20)$. The 
present study demonstrated that overexpression of TASK-1 promoted CD133, OCT-4 and Nanong protein expression in A549 cells. Increased formation of CSC-like populations may result in EMT of cancer cells (21-24). The present study found that overexpression of TASK-1 promotes EMT of A549 cells.

\section{Materials and methods}

Cell line and culture. The A549 NSCLC cell line was purchased from the Cell Bank of the Chinese Academy of Sciences (Shanghai, China) within 3 months of performing the experiments. They were cultured in Dulbecco's modified Eagle's medium supplemented with $10 \%$ fetal bovine serum (both Gibco; Thermo Fisher Scientific, Inc., Waltham, MA, USA) and antibiotics (100 mg/ml penicillin and $100 \mathrm{U} / \mathrm{ml}$ streptomycin) in a $5 \% \mathrm{CO}_{2}$ incubator at $37^{\circ} \mathrm{C}$.

Cell transfection. The pGCMV/EGFP/TASK-1 plasmid and the pGCMV/EGFP/Neo plasmid were constructed (Tiangen Biotech Co., Ltd., Beijing, China). The two plasmids were transfected into A549 NSCLC cells separately using Lipofectamine 2000 transfection reagent (Invitrogen; Thermo Fisher Scientific, Inc.). In order to detect the transfection efficiency of the plasmids, green fluorescent signal was measured by fluorescence microscopy. Subsequent experimentation was performed after $24 \mathrm{~h}$.

Western blot analysis. Total proteins in cells were extracted using protein lysis solution (Tiangen Biotech Co., Ltd.). Protein concentration was measured using a bicinchoninic acid kit (Tiangen Biotech Co., Ltd.). Protein extracts (50 $\mu \mathrm{g} /$ lane) were resolved through $8 \%$ SDS-PAGE, transferred onto polyvinylidene difluoride membranes (Bio-Rad Laboratories, Inc., Hercules, CA, USA) and blocked with 5\% non-fat milk for $1 \mathrm{~h}$ at room temperature. Membranes were probed with antibodies against human TASK-1 (ab135883), vimentin (ab92547), E-cadherin (ab40772), CD133 (ab16518), OCT4 (ab109183), Nanog (ab109250), matrix metalloproteinase (MMP)9 (ab76003), MMP2 (ab92536) or $\beta$-actin (ab8227) (all 1:500; Abcam, Cambridge, MA, USA) at $4^{\circ} \mathrm{C}$ overnight. Membranes were then incubated with anti-rabbit secondary antibodies (1:500; ab218695; Abcam) for $1 \mathrm{~h}$ at room temperature. An enhanced chemiluminescence system (GE Healthcare, Chicago, IL, USA) was used to detect the antibody binding.

Sphere formation assay. Cells were seeded at $2.5 \times 10^{4}$ cells/well on $0.5 \%$ agar pre-coated 6 -well plates. After 1 week, half the medium was exchanged every third day. After a total of 14 days, single spheres were picked and counted (25). Sphere formation efficiency was calculated by dividing the total number of spheres formed by the total number of live cells seeded multiplied by hundred.

MTT assay. To monitor resistance to gefitinib, A549 cells were treated with gefitinib (purity >99\%; AstraZeneca, Cambridge, $\mathrm{UK}$ ) at different concentrations for $24 \mathrm{~h}$. An MTT assay was performed as described previously (2). Data were analyzed with the software Origin 7.5 (OriginLab, Northampton, MA, USA) to fit a sigmodial curve. The $\mathrm{IC}_{50}$ was the gefitinib concentration that reduced the number of viable cells by $50 \%$.
Immunofluorescence staining. Cells seeded on glass coverslips in 6-well plates were fixed in $4 \%$ formaldehyde solution for $30 \mathrm{~min}$ at room temperature and permeabilized with $0.5 \%$ Triton-X-100/PBS. Cells were blocked with 5\% BSA-PBS (BSA from Sigma-Aldrich; Merck KGaA, Darmstadt, Germany) for $1 \mathrm{~h}$ at room temperature and incubated with primary antibodies against E-cadherin (1:500; ab92547; Abcam) or vimentin (1:500; ab40772; Abcam) at $4^{\circ} \mathrm{C}$ overnight. Cells were then incubated with rhodamine- or fluorescein isothiocyanate-conjugated secondary antibodies (1:500; ab150077; Abcam) for $1 \mathrm{~h}$ at room temperature. The coverslips were counterstained with DAPI and imaged under a confocal microscope (TCS SP5; Lecia Microsystems, Wetzlar, Germany).

Wound healing assay. Cells $\left(5 \times 10^{5}\right)$ were seeded onto each 35-mm glass bottom dish (MatTek Co., Ashland, MA, USA) and cultured at $37^{\circ} \mathrm{C}$ with $5 \% \mathrm{CO}_{2}$ for $24 \mathrm{~h}$. The confluent monolayer of cells was wounded with yellow $200 \mu$ l pipette tips (Tiangen Biotech Co., Ltd.). After washing with warm PBS, the cells were incubated in fresh serum-free culture medium. Images of the wounded areas were captured at different time-points with an inverted microscope (Eclipse TE-2000U; Nikon, Tokyo, Japan) equipped with a video camera (DS-U1; Nikon). Results were examined at five randomly selected fields in each field, at x20 magnification. The wound areas were calculated by ImageJ $1.43 \mathrm{~b}$ software (NIH, Bethesda, MD, USA).

Statistical analysis. Values are expressed as the mean \pm standard error of the mean. SPSS 16.0 software (SPSS, Inc., Chicago, IL, USA) was used for the analysis of experimental data. Student's t-test (two-tailed) was used for comparison between two groups. $\mathrm{P}<0.05$ was considered to indicate a statistically significant difference.

\section{Results}

Overexpression of TASK-1 promotes gefitinib resistance. In order to confirm the efficiency of plasmid-mediated TASK-1-expression, A549 cells transfected with TASK-1-expressing plasmid and empty vector were subjected to western blot analysis. The results showed that TASK-1 protein was significantly upregulated in A549 cells transfected with TASK-1-expressing plasmid (Fig. 1A). To further identify whether TASK-1 affected the efficacy of gefitinib in A549 cells, A549 cells transfected with TASK-1-expressing plasmids or empty vector were subjected to an MTT assay (Fig. 1B). The results demonstrated that TASK-1 transformed native, gefitinib-sensitive A549 cells into gefitinib-resistant A549 cells, suggesting that its overexpression induced gefitinib resistance (Fig. 1B).

TASK-1 gives A549 cells CIC-like traits. In order to identify whether TASK-1 affects CIC-like traits in A549 cells, a sphere formation assay was performed to assess the capacity of A549 cells for self renewal, which is associated with CICs and CSCs. The results demonstrated that after 14 days of culture TASK-1-overexpressing cells formed bigger spheres than control cells, indicating markedly increased CIC-like traits provided by the TASK-1-expressing plasmids (Fig. 2A). To 
A

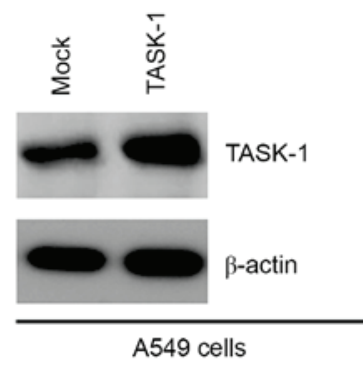

B

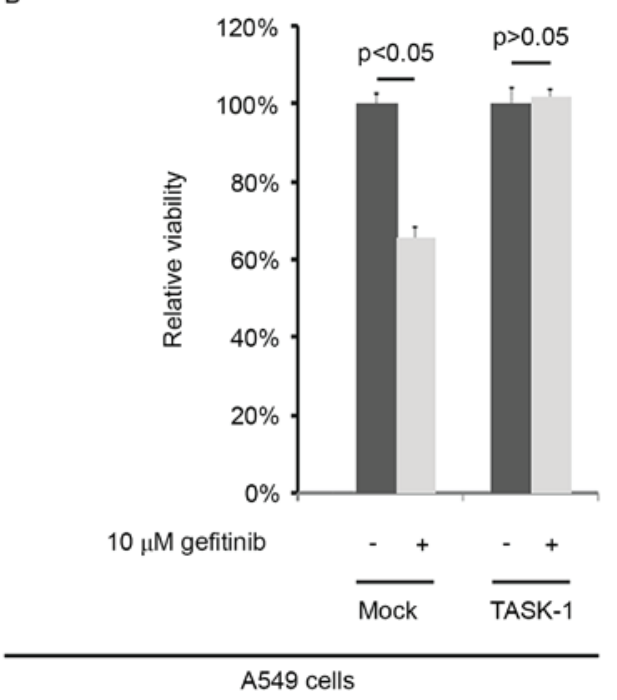

Figure 1. Overexpression of TASK-1 promotes gefitinib resistance. (A) Western blot analysis of TASK-1 in A549 cells. $\beta$-actin was used as a loading control. (B) MTT cell viability assay. A549 cells transfected with TASK-1 expressing plasmids or empty vector (mock) were untreated or treated with gefitinib. Values are expressed as the mean \pm standard error of the mean $(n=3)$. TASK-1, TWIK-related acid-sensitive $\mathrm{K}(+)$.

identify whether TASK-1 regulates CD133, OCT-4 and Nanog protein expression, western blot analysis of A549 cells transfected with TASK-1-expressing plasmids and empty vector was performed. The results revealed that CD133, OCT-4 and Nanog protein were upregulated in A549 cells by TASK-1 (Fig. 2B).

TASK-1 induces EMT in A549 cells. Cell morphological observation revealed that overexpression of TASK-1 induced EMT phenotypes in A549 cells (transition from a cobblestone-like to a spindle-like morphology; Fig. 3A). In order to detect whether TASK-1 affects E-cadherin (epithelial marker) and vimentin (mesenchymal marker) protein, immunofluorescence analysis of A549 cells transfected with TASK-1 and empty vector was performed. It was found that overexpression of TASK-1 promoted vimentin expression and inhibited E-cadherin expression in A549 cells (Fig. 3B). Western blot analysis was also performed to detect E-cadherin and vimentin protein in A549 cells transfected with TASK-1 and empty vector. The results revealed that vimentin protein is upregulated, while E-cadherin is downregulated in A549 cells transfected with TASK-1 overexpression vector (Fig. 3C).

Overexpression of TASK-1 promotes migration of A549 cells. In an attempt to identify the role of TASK-1 in regulating the

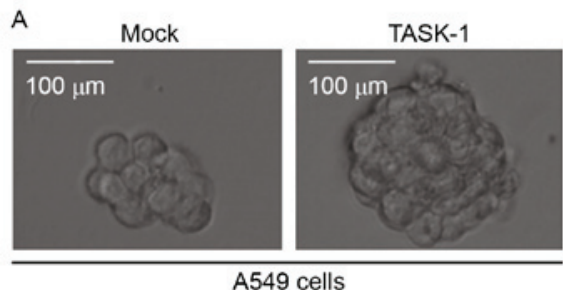

B

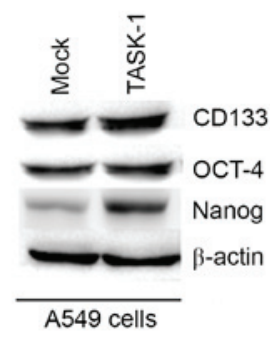

Figure 2. TASK-1 induces formation of CIC phenotypes in A549 cells (A) Sphere growth of A549 cells transfected with TASK-1-expressing plasmids and empty vectors (mock) (scale bar, $100 \mu \mathrm{m}$ ). (B) Western blot analysis of CD133, OCT-4 and Nanog in A549 cells transfected with TASK-1-expressing plasmids and empty vectors (mock). $\beta$-actin was used as a loading control. Representative images of triplicate experiments are shown. TASK-1, TWIK-related acid-sensitive K(+); OCT-4, octamer-binding transcription factor 4.

migration of A549 cells, a would healing assay was performed to detect the migration of A549 cells transfected with TASK-1-expressing plasmid and empty vector. Overexpression of TASK-1 was found to promote the migration in the cells (Fig. 4A). In order to detect whether TASK-1 affects MMP-2 and MMP-9 protein expression, western blot analysis of A549 cells transfected with TASK-1 and empty vector was performed. It was revealed that expression of TASK-1 promotes MMP-2 and MMP-9 expression in A549 cells (Fig. 4B).

\section{Discussion}

NSCLC patients with early-stage disease are treated by surgery, and 30-60\% develop recurrent tumors, which results in mortality $(26,27)$. Chemotherapeutic agents, including gemcitabine, platinum compounds and taxanes, improve survival to a limited extent, but overall survival rates remain low due to recurrence of more aggressive, drug-resistant tumors $(28,29)$. NSCLC in non-smokers predominantly has mutations in EGFR (30); such patients respond well to EGFR inhibitors such as gefitinib, but eventually develop resistance and succumb to the disease (31). The recurrence may be local or metastatic, and commonly occurs after a period of clinical dormancy. Resistance to EGFR inhibitors occurs through various mechanisms, including the appearance of a T790M gatekeeper mutation, expression of the c-Met gene or activation of alternate signaling pathways $(32,33)$. Development of strategies to combat resistance to EGFR inhibitors in NSCLC will provide an immense benefit to a large number of patients (34).

A recent study reported that knockdown of TASK-1 by small interfering RNA significantly enhanced apoptosis and reduced proliferation in A549 cells (18). For the first time, to the best of our knowledge, the present study demonstrated that overexpression of TASK-1 induced gefitinib resistance in 

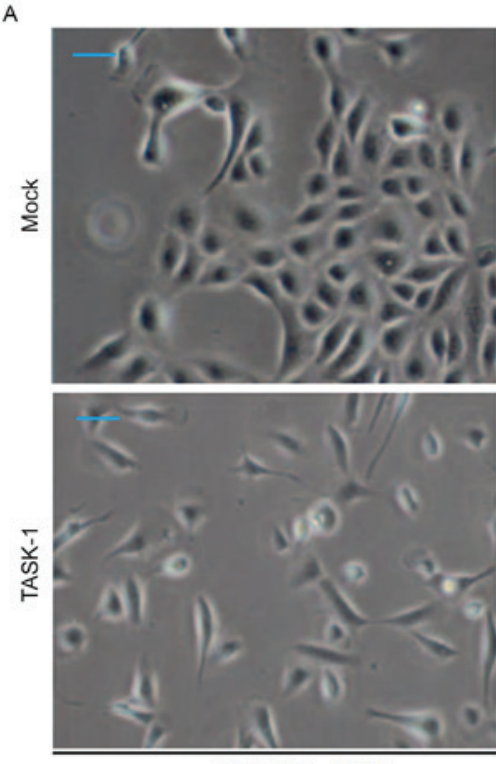

A549 cells (x100)

B Vimentin
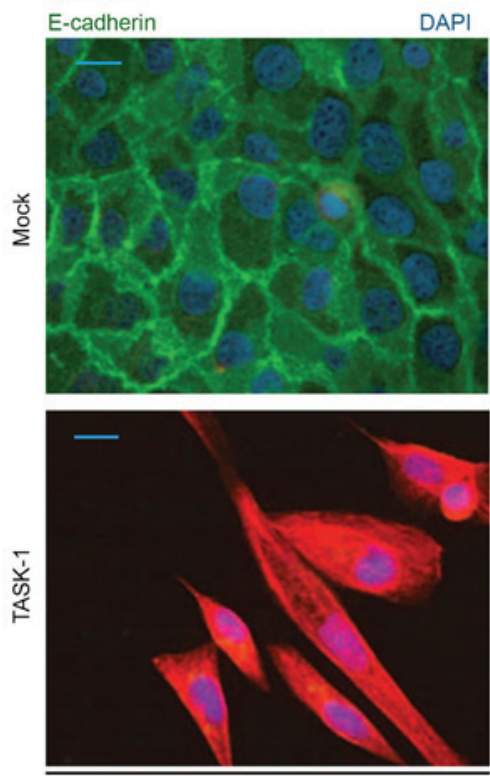

A549 cells ( $x 100)$

C

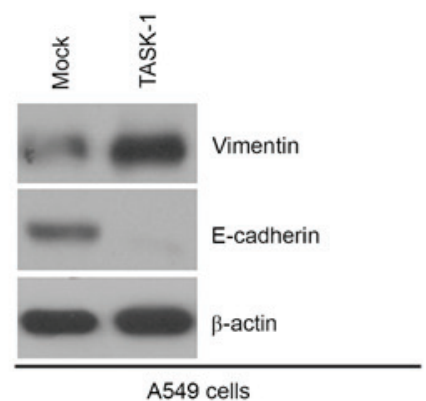

Figure 3. TASK-1 induces epithelial to mesenchymal transition of A549 cells. (A) Phase-contrast microscopy images of A549 cells transfected with TASK-1-expressing plasmids and empty vectors (mock). Scale bar $=100 \mu \mathrm{m}$. (B) Immunofluorescence analyses for vimentin and E-cadherin in A549 cells transfected with TASK-1-expressing plasmids and empty vectors (mock). The nuclei were counterstained with DAPI. Scale bar $=100 \mu \mathrm{m}$. (C) Western blot analysis of vimentin and E-cadherin in A549 cells transfected with TASK-1 expressing plasmids and empty vectors (mock). $\beta$-actin was used as a loading control. Representative images of triplicate experiments are shown. TASK-1, TWIK-related acid-sensitive $\mathrm{K}(+)$.
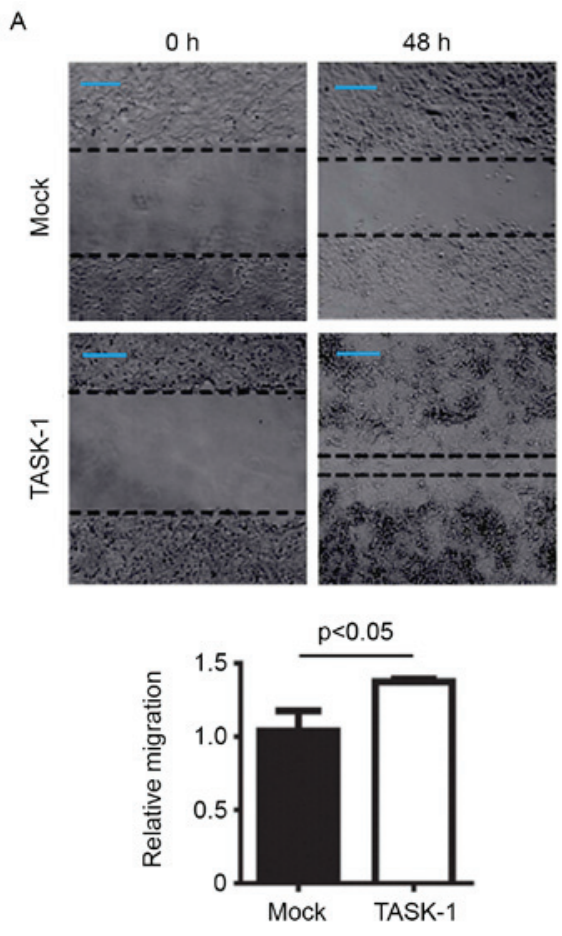

A549 cells

B

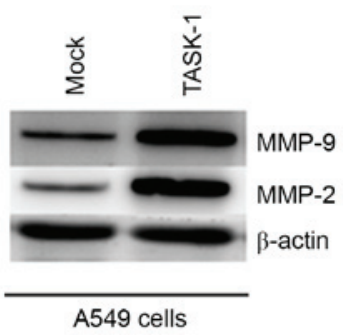

Figure 4. Overexpression of TASK-1 promotes migration of A549 cells (A) Wound-healing assay of A549 cells transfected with TASK-1-expressing plasmids and empty vectors (mock). Images of the cell monolayers were captured directly after scraping and subsequent to $48 \mathrm{~h}$ of incubation. The wound healing rate was quantified by detecting the percentage of wound closure vs. that of the original wound. Values are expressed as the mean \pm standard error of the mean $(\mathrm{n}=3)$. Scale bar $=100 \mu \mathrm{m}$. (B) Western blot analysis of MMP-2 and MMP-9 expression in A549 cells transfected with TASK-1-expressing plasmids and empty vectors (mock). $\beta$-actin was used as a loading control. Representative images of triplicate experiments are shown. MMP, matrix metalloproteinase; TASK-1, TWIK-related acid-sensitive K(+).

A549 cells, implying that TASK-1 may represent a novel target to reverse gefitinib resistance.

Resistance to radiation therapies and conventional chemotherapeutic drugs is a common characteristic of CSCs (35-37). It has been reported that aldehyde dehydrogenase 1 family member A1 (ALDH1A1)-positive CSCs promote gefitinib resistance in lung cancer (17). The present study found that overexpression of TASK-1 promoted A549 cells to adopt CIC-like properties. In addition, overexpression promoted CD133, OCT-4 and Nanog protein expression in A549 cells, indicating that TASK-1 induces the development of CIC-like traits. However, it remains elusive whether TASK-1 promotes the formation of ALDH1A1-positive lung CICs. 
Increased formation of a CIC population may result in EMT of cancer cells and EMT has been shown to contribute to the formation of CIC-like characteristics (38). It has been suggested that the reversal of the EMT phenotype potentially enhances the sensitivity of lung cancer cells to gefitinib (39). Consistent with these results, the present study demonstrated that overexpression of TASK-1 induced EMT of A549 cells. The degradation of ECM and the destruction of basement membrane are prerequisites for tumor infiltration and metastasis (40). Tumor cells must have the ability to degrade ECM and basement membranes, and this degradation process depends on proteolytic enzymes, mainly serine-degrading enzymes, cysteine proteases and MMPs. MMPs are considered to be the most important. MMP2 and MMP9 are important members of the MMP family, encoding $72 \mathrm{KDa}$ gelatinase A and $92 \mathrm{KDa}$ gelatinase $\mathrm{B}$, respectively, which have a partial co-acting substrate that degrades the major constituents of the basement membrane IV type collagen. This is conducive to the migration of tumor cells $(41,42)$.

In conclusion, the results of the present study provided strong molecular evidence demonstrating that TASK-1 promotes gefitinib resistance, EMT and CIC-like properties of NSCLCs. Thus, downregulation of TASK-1 appears to be a novel strategy for the reversal of the EMT, decreasing CIC-like traits and increasing gefitinib sensitivity of NSCLCs.

\section{References}

1. Ding L, Getz G, Wheeler DA, Mardis ER, McLellan MD, Cibulskis K, Sougnez C, Greulich H, Muzny DM, Morgan MB, et al: Somatic mutations affect key pathways in lung adenocarcinoma. Nature 455: 1069-1075, 2008.

2. Sanders HR and Albitar M: Somatic mutations of signaling genes in non-small-cell lung cancer. Cancer Genet Cytogenet 203: 7-15, 2010.

3. Costa DB, Kobayashi S, Tenen DG and Huberman MS: Pooled analysis of the prospective trials of gefitinib monotherapy for EGFR-mutant non-small cell lung cancers. Lung Cancer 58: 95-103, 2007.

4. Miller VA, Riely GJ, Zakowski MF, Li AR, Patel JD, Heelan RT, Kris MG, Sandler AB, Carbone DP, Tsao A, et al: Molecular characteristics of bronchioloalveolar carcinoma and adenocarcinoma, bronchioloalveolar carcinoma subtype, predict response to erlotinib. J Clin Oncol 26: 1472-1478, 2008.

5. Jackman DM, Miller VA, Cioffredi LA, Yeap BY, Jänne PA, Riely GJ, Ruiz MG, Giaccone G, Sequist LV and Johnson BE: Impact of epidermal growth factor receptor and KRAS mutations on clinical outcomes in previously untreated non-small cell lung cancer patients: Results of an online tumor registry of clinical trials. Clin Cancer Res 15: 5267-5273, 2009.

6. Rho JK, Choi YJ, Lee JK, Ryoo BY, Na II, Yang SH, Kim CH and Lee JC: Epithelial to mesenchymal transition derived from repeated exposure to gefitinib determines the sensitivity to EGFR inhibitors in A549, a non-small cell lung cancer cell line. Lung Cancer 63: 219-226, 2009.

7. Li D, Zhang L, Zhou J and Chen H: Cigarette smoke extract exposure induces EGFR-TKI resistance in EGFR-mutated NSCLC via mediating Src activation and EMT. Lung Cancer 93: 35-42, 2016.

8. Grünert S, Jechlinger $M$ and Beug H: Diverse cellular and molecular mechanisms contribute to epithelial plasticity and metastasis. Nat Rev Mol Cell Biol 4: 657-665, 2003.

9. Kalluri R and Neilson EG: Epithelial-mesenchymal transition and its implications for fibrosis. J Clin Invest 112: 1776-1784, 2003.

10. Thiery JP: Epithelial-mesenchymal transitions in development and pathologies. Curr Opin Cell Biol 15: 740-746, 2003.

11. Thiery JP: Epithelial-mesenchymal transitions in tumour progression. Nat Rev Cancer 2: 442-454, 2002.

12. Pine SR, Marshall B and Varticovski L: Lung cancer stem cells. Dis Markers 24: 257-266, 2008.
13. Eramo A, Lotti F, Sette G, Pilozzi E, Biffoni M, Di Virgilio A, Conticello C, Ruco L, Peschle C and De Maria R: Identification and expansion of the tumorigenic lung cancer stem cell population. Cell Death Differ 15: 504-514, 2008.

14. Kim CF, Jackson EL, Woolfenden AE, Lawrence S, Babar I, Vogel S, Crowley D, Bronson RT and Jacks T: Identification of bronchioalveolar stem cells in normal lung and lung cancer. Cell 121: 823-835, 2005.

15. Jiang F, Qiu Q, Khanna A, Todd NW, Deepak J, Xing L, Wang H, Liu Z, Su Y, Stass SA and Katz RL: Aldehyde dehydrogenase 1 is a tumor stem cell-associated marker in lung cancer. Mol Cancer Res 7: 330-338, 2009.

16. Dean M, Fojo T and Bates S: Tumour stem cells and drug resistance. Nat Rev Cancer 5: 275-284, 2005.

17. Huang CP, Tsai MF, Chang TH, Tang WC, Chen SY, Lai HH, Lin TY, Yang JC, Yang PC, Shih JY and Lin SB: ALDH-positive lung cancer stem cells confer resistance to epidermal growth factor receptor tyrosine kinase inhibitors. Cancer Lett 328: 144-151, 2013.

18. Leithner K, Hirschmugl B, Li Y, Tang B, Papp R, Nagaraj C, Stacher E, Stiegler P, Lindenmann J, Olschewski A, et al: TASK-1 regulates apoptosis and proliferation in a subset of non-small cell lung cancers. PLoS One 11: e0157453, 2016.

19. Chen YC, Hsu HS, Chen YW, Tsai TH, How CK, Wang CY, Hung SC, Chang YL, Tsai ML, Lee YY, et al: Oct-4 expression maintained cancer stem-like properties in lung cancer-derived CD133-positive cells. PLoS One 3: e2637, 2008.

20. Chiou SH, Wang ML, Chou YT, Chen CJ, Hong CF, Hsieh WJ, Chang HT, Chen YS, Lin TW, Hsu HS and Wu CW: Coexpression of Oct4 and nanog enhances malignancy in lung adenocarcinoma by inducing cancer stem cell-like properties and epithelial-mesenchymal transdifferentiation. Cancer Res 70: 10433-10444, 2010.

21. Kurrey NK, Jalgaonkar SP, Joglekar AV, Ghanate AD, Chaskar PD, Doiphode RY and Bapat SA: Snail and slug mediate radioresistance and chemoresistance by antagonizing p53-mediated apoptosis and acquiring a stem-like phenotype in ovarian Cancer cells. Stem Cells 27: 2059-2068, 2009.

22. Mani SA, Guo W, Liao MJ, Eaton EN, Ayyanan A, Zhou AY, Brooks M, Reinhard F, Zhang CC, Shipitsin M, et al: The epithelial-mesenchymal transition generates cells with properties of stem cells. Cell 133: 704-715, 2008.

23. Morel AP, Lièvre M, Thomas C, Hinkal G, Ansieau S and Puisieux A: Generation of breast cancer stem cells through epithelial-mesenchymal transition. PLoS One 3: e2888, 2008.

24. Santisteban M, Reiman JM, Asiedu MK, Behrens MD, Nassar A, Kalli KR, Haluska P, Ingle JN, Hartmann LC, Manjili MH, et al: Immune-induced epithelial to mesenchymal transition in vivo generates breast cancer stem cells. Cancer Res 69: 2887-2895, 2009.

25. Tiran V, Stanzer S, Heitzer E, Meilinger M, Rossmann C, Lax S, Tsybrovskyy O, Dandachi N and Balic M: Genetic profiling of putative breast cancer stem cells from malignant pleural effusions. PLoS One 12: e0175223, 2017.

26. Demicheli R, Fornili M, Ambrogi F, Higgins K, Boyd JA, Biganzoli E and Kelsey CR: Recurrence dynamics for non-small-cell lung cancer: Effect of surgery on the development of metastases. J Thorac Oncol 7: 723-730, 2012.

27. Senthi S, Lagerwaard FJ, Haasbeek CJ, Slotman BJ and Senan S: Patterns of disease recurrence after stereotactic ablative radiotherapy for early stage non-small-cell lung cancer: A retrospective analysis. Lancet Oncol 13: 802-809, 2012.

28. Sève P and Dumontet C: Chemoresistance in non-small cell lung cancer. Curr Med Chem Anticancer Agents 5: 73-88, 2005.

29. Lara PN Jr, Lau DH and Gandara DR: Non-small-cell lung cancer progression after first-line chemotherapy. Curr Treat Options Oncol 3: 53-58, 2002.

30. Sun S, Schiller JH and Gazdar AF: Lung cancer in never smokers-a different disease. Nat Rev Cancer 7: 778-790, 2007.

31. Brugger W and Thomas M: EGFR-TKI resistant non-small cell lung cancer (NSCLC): New developments and implications for future treatment. Lung Cancer 77: 2-8, 2012.

32. Nurwidya F, Takahashi F, Murakami A, Kobayashi I, Kato M, Shukuya T, Tajima K, Shimada N and Takahashi K: Acquired resistance of non-small cell lung cancer to epidermal growth factor receptor tyrosine kinase inhibitors. Respir Investig 52: 82-91, 2014.

33. Wangari-Talbot $\mathbf{J}$ and Hopper-Borge E: Drug resistance mechanisms in non-small cell lung carcinoma. J Can Res Updates 2: 265-282, 2013. 
34. Chong CR and Jänne PA: The quest to overcome resistance to EGFR-targeted therapies in cancer. Nat Med 19: 1389-1400, 2013.

35. Zhou BB, Zhang H, Damelin M, Geles KG, Grindley JC and Dirks PB: Tumour-initiating cells: Challenges and opportunities for anticancer drug discovery. Nat Rev Drug Discov 8: 806-823, 2009.

36. Frank NY, Schatton T and Frank MH: The therapeutic promise of the cancer stem cell concept. J Clin Invest 120: 41-50, 2010.

37. Wang J, Wakeman TP, Lathia JD, Hjelmeland AB, Wang XF, White RR, Rich JN and Sullenger BA: Notch promotes radioresistance of glioma stem cells. Stem Cells 28: 17-28, 2010.

38. Polyak K and Weinberg RA: Transitions between epithelial and mesenchymal states: Acquisition of malignant and stem cell traits. Nat Rev Cancer 9: 265-273, 2009.
39. Xie M, Zhang L, He CS, Xu F, Liu JL, Hu ZH, Zhao LP and Tian Y: Activation of notch-1 enhances epithelial-mesenchymal transition in gefitinib-acquired resistant lung cancer cells. J Cell Biochem 113: 1501-1513, 2012.

40. Hirvonen R, Talvensaari-Mattila $A$, Pääkkö $P$ and Turpeenniemi-Hujanen T: Matrix metalloproteinase-2 (MMP-2) in T(1-2)N0 breast carcinoma. Breast Cancer Res Treat 77: 85-91, 2003.

41. Kalhori V and Törnquist K: MMP2 and MMP9 participate in S1P-induced invasion of follicular ML-1 thyroid cancer cells. Mol Cell Endocrinol 404: 113-122, 2015.

42. Liu B, Cui J, Sun J, Li J, Han X, Guo J, Yi M, Amizuka N, Xu X and Li M: Immunolocalization of MMP9 and MMP2 in osteolytic metastasis originating from MDA-MB-231 human breast cancer cells. Mol Med Rep 14: 1099-1106, 2016. 